\title{
Detektion asymptomatischer angeborener Herzfehler
}

\section{Pulsoxymetriescreening bei Neugeborenen}

\section{Originalpublikation \\ Thangaratinam S, Brown K, Za- mora J et al (2012) Pulse oxime- try screening for critical congeni- tal heart defects in asymptoma- tic newborn babies: a systematic review and meta-analysis. Lancet May 1. [Epub ahead of print]. DOI 10.1016/S0140-6736(12)60107}

\section{Hintergrund}

Seit einigen Jahren wird die Pulsoxymetrie zur Erkennung angeborener Herzfehler propagiert, ihre Eignung als Screeningmethode ist jedoch umstritten.

\section{Studie}

Für die vorliegende Arbeit wurden 13 Studien mit insgesamt knapp 230.000 Neugeborenen einer Metaanalyse unterzogen. Kriterien waren u. a. die Definition eines kritischen angeborenen Herzfehlers als lebensbedrohlicher Defekt, der innerhalb von 28 Lebenstagen zum Tode führt oder einer invasiven Prozedur (meist Operation) bedarf. Hierzu gehören hypoplastisches Linksherz, Pulmonalatresie mit intaktem Ventrikelseptum, Transposition der großen Arterien, unterbrochener Aortenbogen sowie Koarktation der Aorta, Aorten- und Pulmonalklappenstenose, Fallot-Tetralogie, Pulmonalatresie mit Ventrikelseptumdefekt und totale Lungenvenenfehlmündung.

Monatsschr Kinderheilkd 2012 • 160:617-617 DOI 10.1007/s00112-012-2678-5

\section{Redaktion:}

O. Bodamer (Miami), J. Freihorst (Aalen),

R. Kerbl (Leoben), G. Krandick (Oberhaching)
Alle Kinder waren zum Zeitpunkt der Untersuchung asymptomatisch, in 9 der 13 Studien wurden Kinder mit pränatal diagnostiziertem Herzfehler ausgeschlossen. Die Messwerte wurden am ersten Lebenstag (6 Studien) oder im Alter von mehr als $24 \mathrm{~h}$ erhoben.

In $60 \%$ der Studien wurde die Pulsoxymetrie ausschließlich am Fuß, in den anderen Arbeiten zusätzlich an der rechten Hand durchgeführt. Positive Messergebnisse wurden mittels Echokardiographie überprüft, negative Resulate durch Auswertung von Mortalitätsdaten, Anomalienregistern und klinischem Follow-up. Über alle Studien fanden sich eine Sensitivität von $76,5 \%$ (95\%-Konfidenzintervall $67,7-83,5)$ und eine Spezifität von $99,9 \%$ (95\%-Konfidenzintervall 99,7-99,9). Falsch-positive Werte gab es bei Messung am ersten Lebenstag in $0,5 \%$ (95\%-Konfidenzintervall 0,29$0,86)$ und nach Ablauf von $24 \mathrm{~h}$ in $0,05 \%$ (95\%-Konfidenzintervall $0,02-0,12, \mathrm{p}-0,0017) \mathrm{der}$ Fälle.

\section{Ergebnisse}

Die Autoren kommen zu dem Schluss, dass ein generelles Screening auf kongenitale Herzfehler bei asymptomatischen Neugeborenen gerechtfertigt und sinnvoll ist. In Anbetracht der großen Zahl ausgewerteter Patienten sind bei weiterer Forschung auf diesem Gebiet keine substanziell anderen Ergebnisse zu erwarten.

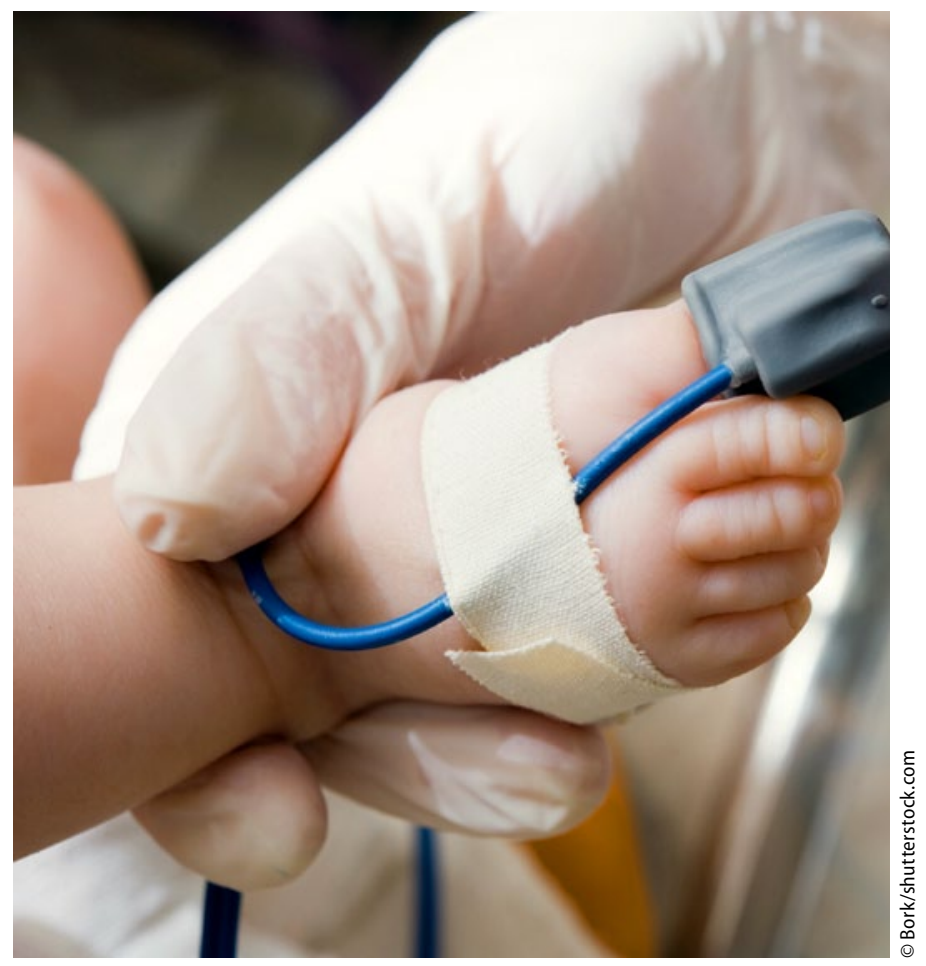

Abb.1 $\Delta$ Bei jedem Neugeborenen eine Pulsoxymetrie erfolgen, wobei die relativ mäßige Sensitivität zu beachten bleibt

\section{Kommentar}

In Zeiten ausgefeilter pränataler Diagnostik und umfassender Vorsorgeprogramme erscheint es unwahrscheinlich, ist jedoch eine Tatsache, dass Neugeborene mit z. B. ductusabhängigen Vitien undiagnostiziert die Klinik verlassen. Manifestation des Herzfehlers mit Kreislaufversagen und kritischem klinischem Zustand verschlechtert in diesen Fällen die Prognose. In Anbetracht der Datenlage sollte bei jedem Neugeborenen eine Pulsoxymetrie erfolgen, wobei die relativ mäßige Sensitivität zu beachten bleibt. Der Zeitpunkt der Messung muss für ein Screeningprogramm noch genau festgelegt werden, wird aber in An- betracht der immer kürzeren postnatalen Verweildauer in der Klinik eher innerhalb der ersten 24 Lebensstunden des Neugeborenen liegen.

Dr. G. Krandick

Jugendmedizin

Tisinstraße 56a

82041 Deisenhofen

mail@dr-krandick.de

\section{Korrespondenzadresse}

Facharzt für Kinder- und 